ISSN 0103-5150

Fisioter. Mov., Curitiba, v. 26, n. 3, p. 569-578, jul./set. 2013

Licenciado sob uma Licença Creative Commons

\title{
Preferência por lado mastigatório e amplitude de movimento cervical em laringectomizados totais
}

\author{
Relationship between chewing side preference and \\ range of motion in total laryngectomy patients
}

\author{
Thais Myrian Aragão Mélo ${ }^{[a]}$, Celina Cordeiro de Carvalho ${ }^{[b]}$, \\ Gerlane Karla Bezerra Oliveira Nascimento ${ }^{[\mathrm{cl}}$, Klyvia Juliana Rocha de Moraes ${ }^{[\mathrm{d}]}$, \\ Hilton Justino da Silva ${ }^{[e]}$
}

[a] Fisioterapeuta, mestre em Patologia pela Universidade Federal de Pernambuco (UFPE), Recife, PE - Brasil, e-mail: thaismaragao@hotmail.com

[b] Fisioterapeuta, doutora em Neuropsiquiatria e Ciências do Comportamento pela Universidade Federal de Pernambuco (UFPE), docente do curso de Fisioterapia da Faculdade Estácio do Recife, Recife, PE - Brasil, e-mail: celinacordeiro@hotmail.com

[c] Fonoaudióloga, mestre em Patologia pela Universidade Federal de Pernambuco (UFPE), Fonoaudióloga da Secretaria de Saúde do Estado da Paraíba, João Pessoa, PB - Brasil, e-mail: gerlane_fono@hotmail.com

[d] Fisioterapeuta, mestre em Patologia pela Universidade Federal de Pernambuco (UFPE), docente do curso de Fisioterapia da Universidade Salgado de Oliveira (Universo), Recife, PE - Brasil, e-mail: klyviaj@hotmail.com

[e] Fonoaudiólogo, doutor em Nutrição pela Universidade Federal de Pernambuco (UFPE), docente do curso de Fonoaudiologia da Universidade Federal de Pernambuco (UFPE), Recife, PE - Brasil, e-mail: hiltonfono@hotmail.com

\section{Resumo}

Introdução: A laringectomia total leva a aderências, dor, encurtamentos musculares e deficit na mobilidade de pescoço. Interfere em funções do sistema estomatognático; exemplo importante é a mastigação. Objetivo: Certificar-se da existência de uma possível predominância por lado mastigatório em laringectomizados totais e de sua relação com a diminuição das amplitudes dos movimentos de cabeça e pescoço. Materiais e métodos: A população de estudo foi constituída de 18 voluntários adultos, divididos em dois grupos: grupo dos laringectomizados totais e grupo controle. Todos os voluntários foram submetidos a uma avaliação goniométrica da amplitude de movimento de cabeça para flexão, extensão, inclinação e rotação, e uma mastigatória para identificar a existência de preferência por um lado mastigatório. Resultados: Houve preferência por lado mastigatório com predomínio à esquerda em laringectomizados totais. Na avaliação goniométrica, o 
movimento de extensão ativa e passiva foi o único com amplitude de movimento reduzida de forma significativa em laringectomizados totais quando comparados ao grupo controle. Relacionando o lado de preferência mastigatória com os achados goniométricos, quando ocorreu à direita, o movimento com amplitude limitada significativamente foi somente o de extensão ativa e passiva. Já quando a preferência ocorreu à esquerda, houve redução do ângulo de extensão passiva e da inclinação para a direita, tanto ativa quanto passiva. Conclusão: Os laringectomizados totais, apresentaram preferência por lado mastigatório à esquerda, acompanhada de redução significativa da amplitude de movimento somente da extensão passiva e da inclinação para a direita, tanto ativa quanto passiva.

Palavras-chave: Laringectomia. Mastigação. Amplitude de movimento articular. Cabeça. Pescoço.

\section{Abstract}

Introduction:Total laryngectomy leads to adhesions, pain, muscle shortening and deficit in mobility of the neck. Interfere with functions of the stomatognathic system; chewing is an important example. Objective: Verify a possible predominance of chewing side in laryngectomized and the decrease related in the amplitude of movements of head and neck. Materials and methods: The study population consisted of 18 adult volunteers were divided into two groups: the total laryngectomy group and control group. All subjects underwent an evaluation of goniometric range of motion of head flexion, extension, tilt and rotation, and a masticatory to identify the existence of a preference for one side chewing. Results: There was chewing side preference for predominantly left in total laryngectomy. In the goniometric evaluation, the extension movement was active and passive range of motion with only reduced significantly in total laryngectomy compared to the control group. Relating the masticatory preference side with the goniometric findings, when it occurred to the right, the movement amplitude was significantly limited in length only the active and passive. But when the choice was left, there was a reduction of the passive extension angle and tilt to the right, either actively or passively. Conclusion: The total laryngectomy showed a preference for left hand chewing and is accompanied by a significant reduction in the amplitude of movement and only the passive extension of the inclination to the right, both active and passive.

Keywords: Laryngectomy. Chewing. Range of motion articular. Head. Neck.

\section{Introdução}

Para o tratamento do câncer de laringe existem diferentes intervenções terapêuticas, dependendo do estadiamento, da localização e da extensão do tumor. Sequelas do tratamento comprometem a qualidade de vida do paciente de forma temporária ou permanente $(1,2)$, com alterações anatômicas, fisiológicas / funcionais e psicossociais $(3,4)$.

0 início desse tipo de câncer comumente é insidioso, lento e silencioso (5). Por isso, muitos desses tumores malignos são diagnosticados quando já se encontram em fase avançada (3), momento em que o tratamento é, quase sempre, a associação de laringectomia total com radioterapia $(6,7)$ e/ou quimioterapia $(8,9)$. Com a laringectomia total são removidas as estruturas que produzem o som laríngeo. A parte superior da faringe é suturada junto à base da língua e a traqueia é suturada junto à pele da base do pescoço onde é realizada a traqueostomia, a partir da qual a respiração tornar-se-á definitiva (5). Assim, os sistemas digestório e respiratório tornam-se separados e independentes (10).

A laringectomia total desencadeia, então, alterações em estruturas responsáveis por realizar funções básicas do sistema estomatognático: mastigação, deglutição, fonação e respiração (3). 0 procedimento também resulta em deficit na mobilidade de pescoço (4), encurtamentos musculares, dor e aderências (11).

No sistema estomatognático, é a mastigação que desempenha o papel mais importante (12), porém o equilíbrio da função mastigatória depende da alternância do alimento entre os lados da boca, intercalando assim, o período de trabalho e de repouso. A presença de uma mastigação unilateral tem como consequência a assimetria funcional dos músculos e movimentos mandibulares (13).

Estudos já vêm reportando a ligação funcional entre o sistema motor mandibular e o cervical e sua 
interdependência $(14,15)$. A compreensão do comportamento da amplitude de movimento de cabeça e pescoço nessa população e sua relação com uma provável mastigação unilateral pode contribuir para o aumento do conhecimento em relação às repercussões musculoesqueléticas da laringectomia total com consequente melhoria nas alternativas de tratamento. 0 método mais utilizado na prática clínica para aferição da amplitude de movimento em determinada articulação é a goniometria (16). Algumas das vantagens são o baixo custo, o fácil manuseio (17) e a alta confiabilidade intrassessão $(16,18)$.

Objetiva-se por este estudo verificar a possível existência da predominância de um lado mastigatório em laringectomizados totais e sua relação com a diminuição das amplitudes dos movimentos da cabeça e do pescoço.

\section{Materiais e métodos}

Este estudo foi aprovado pelo Comitê de Ética e Pesquisa do Hospital de Câncer de Pernambuco, sob o número $57 / 2010$, e todos os voluntários, após serem orientados sobre o que propunha o projeto e terem concordado em participar, assinaram o Termo de Consentimento Livre e Esclarecido, conforme Resolução MS/CNS/ CNEP n. 196/96.

A população de estudo foi composta por 18 voluntários adultos (12 do gênero masculino e 6 do feminino), selecionados por conveniência, divididos em dois grupos: grupo laringectomizados totais LT ( $\mathrm{n}=9$ ); e grupo controle, formado por familiares com mesma faixa etária e realidade socioeconômica dos laringectomizados totais $(\mathrm{n}=9)$ ).

Os critérios de inclusão do grupo LT foram: estar em pós-operatório de cirurgia eletiva de laringectomia total no Hospital de Câncer de Pernambuco (HCP); estar em atendimento fonoaudiológico no ambulatório de Fonoaudiologia do HCP; ter realizado radioterapia pós-cirúrgica; ter ao menos 40 anos; ter capacidade para mastigação de alimentos sólidos. Foram excluídos desse grupo os que haviam se submetido a: cirurgia prévia em região de cabeça e pescoço; outro tipo de laringectomia; traqueostomia prévia à laringectomia total. Também foram excluídos os que utilizaram via de alimentação alternativa prévia à laringectomia total ou presente no momento da aplicação do teste; que passaram por complicações pós-operatórias como fístula faringocutânea, deiscências e necrose de tecido; usuários de próteses dentárias parciais ou totais mal adaptadas; comprometimento do nervo acessório espinhal; pacientes com dificuldade na compreensão de ordens simples ou doenças neurológicas, neuromusculares ou neurodegenerativas comprovadas anteriormente; voluntários já submetidos ou que estivessem realizando algum tipo de tratamento fisioterapêutico; e com acometimento por disfunção temporomandibular (DTM).

No grupo controle, foram incluídos indivíduos com mesma faixa etária dos LT (no mínimo 40 anos); de mesmo grupo familiar e mesma realidade socioeconômica. Os critérios de exclusão foram: ter se submetido a cirurgia prévia em região de cabeça e pescoço; ter utilizado via de alimentação alternativa no momento da avaliação; apresentar comprometimento do nervo acessório espinhal; ter dificuldade na compreensão de ordens simples ou doenças neurológicas, neuromusculares ou neurodegenerativas comprovadas; ser voluntário já submetido a algum tipo de tratamento fisioterapêutico e/ou fonoaudiológico; ter acometimento por disfunção temporomandibular (DTM) e distúrbios comprovados que interfiram na coluna cervical.

Todos os voluntários foram submetidos a uma avaliação goniométrica; foi igualmente realizada avaliação para identificar o lado de preferência mastigatória. Para ambas avaliações, os voluntários se posicionaram confortavelmente em uma cadeira, com a coluna dorso-lombar apoiada no encosto da cadeira e os pés apoiados no chão na posição neutra. Tanto o quadril quanto os joelhos e tornozelos foram posicionados com $90^{\circ}$ de flexão.

Um fonoaudiólogo avaliou a mastigação dos indivíduos da pesquisa a fim de observar a preferência por lado mastigatório, seguindo o protocolo elaborado por Nascimento (19).

0 voluntário foi posicionado sentado confortavelmente em uma cadeira e a ele foi solicitado que mastigasse fragmentos de pão do tipo francês. Cada fragmento de pão teve o volume equivalente à mordida habitual de cada voluntário para que não houvesse maiores interferências no ato mastigatório. A avaliação desse processo ocorreu durante os primeiros 30 segundos do ato mastigatório.

Os participantes foram filmados durante o ato mastigatório com uma filmadora digital (Sony Digital Hand Cam; VCR TRV 130 NTSC), a qual foi fixada em um tripé com distância de $1,5 \mathrm{~m}$ em relação à cadeira e com altura ao nível da face do voluntário; os dados foram gravados em um cartão de memória da própria filmadora e depois transferidos para um DVD. 
A partir da análise das gravações, a mastigação habitual de cada indivíduo foi caracterizada segundo a predominância por lado mastigatório.

A análise da amplitude de movimento (ADM) do segmento cervical da coluna vertebral, ativa e passivamente foi realizada por um fisioterapeuta, utilizando a goniometria (goniômetro analógico, CARCI - Indústria e Comércio de Aparelhos Cirúrgicos e Ortopédicos Ltda.) e seguindo o protocolo elaborado por Moraes (20). Os movimentos cervicais avaliados foram flexão/extensão, rotação e inclinação para os lados direito e esquerdo.

Os voluntários se posicionaram na cadeira, como já mencionado, com as mãos apoiadas sobre as coxas e o olhar para frente. Para a mensuração dos movimentos de flexão e extensão da cabeça, o eixo do goniômetro foi posicionado sobre o conduto auditivo externo, braço fixo em direção ao teto (alinhado com a linha média vertical da cabeça) e braço móvel alinhado com a ponta do nariz. Para avaliar o movimento de inclinação lateral para a direita e para a esquerda, o eixo do goniômetro foi posicionado no processo espinhoso de C7 com o braço fixo alinhado com os demais processos espinhos abaixo daquele segmento e o braço móvel alinhado com ápice posterior da cabeça. Já para avaliação da ADM da rotação da cabeça para direita e para a esquerda, o eixo do goniômetro foi posicionado no vértex da cabeça, o braço fixo alinhado ao acrômio e o braço móvel à ponta do nariz (21).

Para melhor apresentação dos resultados, primeiramente, o grupo dos laringectomizados totais foi comparado ao controle, para identificar possíveis diferenças nas avaliações goniométricas e de preferência por lado mastigatório entre os grupos. Numa segunda fase, essa comparação foi realizada em cada grupo, para observação de diferenças de amplitude de movimento (goniométricas) em relação à preferência mastigatória.

As variáveis quantitativas, em escala intervalar ou de razões, foram resumidas com os parâmetros da Estatística Descritiva, incluindo média, erro padrão da média, com discriminação dos intervalos de confiança em nível de 95\%, e limites de variação. As diferenças de médias foram submetidas ao teste não paramétrico de Mann-Whitney para comparação de amostras não pareadas, considerando casos e controles, com nível de significância de 0,05 para rejeição da hipótese nula de igualdade de médias.

As variáveis qualitativas, em escala nominal ou ordinal, foram resumidas em distribuição de frequências absolutas e relativas.

O programa utilizado para digitação dos dados e aquisição dos cálculos estatísticos foi o SPSS (Statistical Package for the Social Sciences), na versão 17.0.

\section{Resultados}

De acordo com os resultados obtidos na avaliação fonoaudiológica, a preferência por um lado mastigatório foi observada nos dois grupos. Tanto no LT quanto no controle, a predominância pelo lado direito ocorreu em quatro indivíduos $(44,4 \%)$. Consequentemente, o percentual de voluntários com preferência mastigatória à esquerda, para ambos os grupos, igualou-se a 55,6\% (Tabela 1 ).

Com relação aos resultados da goniometria do segmento cervical (avaliada na forma passiva e ativa) relativos aos movimentos de flexão, extensão, rotação e inclinação, o LT apresentou menor média do ângulo de flexão, tanto na forma ativa quanto na passiva, e maior variabilidade em relação ao controle; porém, os dados não alcançaram significância estatística. A redução da extensão ativa e passiva do segmento cervical do LT foi mais acentuada quando comparada à do controle ( $p<0,001$; Tabela 2).

A amplitude de movimento da rotação foi mais restrita à direita do que à esquerda, na mensuração ativa, já que o intervalo de variação foi o de menor amplitude $\left(26^{\circ}\right.$ a $\left.48^{\circ}\right)$ em relação à aferição

Tabela 1 - Distribuição dos voluntários segundo o lado de preferência mastigatório

\begin{tabular}{lcc}
\hline Lado preferencial de mordida & Frequência & Percentual \\
\hline Direita & 8 & 44,4 \\
Esquerda & 10 & 55,6 \\
Total & 18 & 100,0 \\
\hline
\end{tabular}

Fonte: Dados da pesquisa. 
Tabela 2 - Distribuição de médias, intervalos de confiança e intervalo de variação das avaliações goniométricas de laringectomizados e controles

\begin{tabular}{|c|c|c|c|c|c|c|c|c|}
\hline \multirow{2}{*}{ Parâmetros } & & \multicolumn{3}{|c|}{ Casos (graus) } & \multicolumn{3}{|c|}{ Controles (graus) } & \multirow[b]{2}{*}{$\mathrm{p}$} \\
\hline & & $\begin{array}{c}\text { Média } \pm \\
\text { Erro padrão }\end{array}$ & IC95\% & Variação & $\begin{array}{c}\text { Média } \pm \\
\text { Erro padrão }\end{array}$ & IC95\% & Variação & \\
\hline \multicolumn{9}{|l|}{ Flexão } \\
\hline Ativa & & $20,89 \pm 3,58$ & $12,64-29,14$ & $10-38$ & $24,44 \pm 2,38$ & $18,97-29,92$ & $12-34$ & 0,420 \\
\hline Passiva & & $22,89 \pm 3,85$ & $14,02-31,76$ & $10-44$ & $28,44 \pm 2,35$ & $23,02-33,87$ & $16-36$ & 0,236 \\
\hline \multicolumn{9}{|l|}{ Extensão } \\
\hline Ativa & & $21,00 \pm 3,40$ & $13,16-28,84$ & $10-45$ & $39,78 \pm 2,12$ & $34,89-44,67$ & $26-46$ & $<0,001$ \\
\hline Passiva & & $22,11 \pm 3,43$ & $14,21-30,01$ & $10-45$ & $42,67 \pm 2,00$ & $38,05-47,28$ & $30-48$ & $<0,001$ \\
\hline \multicolumn{9}{|l|}{ Rotação } \\
\hline \multirow{2}{*}{ à direita } & ativa & $37,33 \pm 2,69$ & $31,14-43,53$ & $26-48$ & $41,00 \pm 1,32$ & $37,95-44,05$ & $34-46$ & 0,239 \\
\hline & passiva & $40,89 \pm 3,45$ & $32,93-48,84$ & $26-54$ & $45,22 \pm 1,15$ & $42,57-47,88$ & $40-52$ & 0,262 \\
\hline \multirow{2}{*}{ à esquerda } & à ativa & $42,89 \pm 3,23$ & $35,43-50,35$ & $26-54$ & $48,78 \pm 2,15$ & $43,83-53,73$ & $38-58$ & 0,149 \\
\hline & passiva & $45,56 \pm 3,16$ & $38,27-52,84$ & $28-56$ & $52,56 \pm 2,02$ & $47,89-57,22$ & $41-60$ & 0,080 \\
\hline \multicolumn{9}{|l|}{ Inclinação } \\
\hline \multirow{2}{*}{ à direita } & ativa & $26,11 \pm 3,79$ & $17,38-34,85$ & $10-47$ & $29,44 \pm 1,80$ & $25,29-33,60$ & $22-38$ & 0,438 \\
\hline & passiva & $27,22 \pm 3,74$ & $18,60-35,85$ & $12-47$ & $31,56 \pm 1,76$ & $27,50-35,61$ & $24-40$ & 0,310 \\
\hline \multirow{2}{*}{ à esquerda } & ativa & $27,67 \pm 3,82$ & $18,85-36,48$ & $12-47$ & $29,67 \pm 2,72$ & $23,40-35,93$ & $18-45$ & 0,675 \\
\hline & passiva & $29,00 \pm 3,75$ & $20,35-37,65$ & $12-47$ & $33,33 \pm 2,49$ & $27,58-39,09$ & $24-48$ & 0,350 \\
\hline
\end{tabular}

Fonte: Dados da pesquisa.

Nota: *Valores de p calculados com o teste não paramétrico de Mann-Whitney para comparação de médias de amostras não pareadas.

em condição passiva $\left(26^{\circ}\right.$ a $\left.54^{\circ}\right)$. No entanto, essas diferenças não alcançaram significância estatística. $\mathrm{Na}$ inclinação lateral, constatou-se redução de movimentação tanto à direita quanto à esquerda, em ambas as condições (ativa e passiva), mas a redução da amplitude de movimento foi maior na inclinação à direita especialmente quando realizada de forma ativa. Essas diferenças também não foram significantes, ainda que houvesse grande amplitude.

Limitações maiores de movimentação relacionadas à flexão e extensão no grupo de laringectomizados podem ser observadas no Gráfico 1, representado pelas amplitudes das aferições goniométricas dos grupos de laringectomizados totais e controle.

De acordo com os achados goniométricos em relação à preferência por lado mastigatório, foram observadas reduções das amplitudes de movimento no LT quando comparado ao controle, principalmente nos movimentos de flexão e extensão, e menos acentuadamente na inclinação e na rotação da cabeça, naqueles com preferência mastigatória à direita (Tabela 3).
De todas as alterações, as diminuições da ADM na extensão ativa e passiva foram os únicos parâmetros que atingiram significância estatística ( $p<0,020$ e p $<0,019$, respectivamente) para indivíduos com predomínio de ciclos mastigatórios à direita. Observa-se que o limite superior do ângulo de extensão da cabeça do grupo LT aproxima-se do limite inferior da mobilidade do grupo controle, denotando perda significativa (Tabela 3).

Com a análise da Tabela 4, constata-se que, quando a preferência de mastigação era à esquerda, a redução do ângulo de extensão passiva e da inclinação para a direita, tanto ativa quanto passiva, ocorria, havendo significância nessas diferenças em relação ao grupo controle.

\section{Discussão}

A partir dos resultados encontrados neste estudo, foi possível observar que existe uma predominância, tanto em laringectomizados totais quanto em indivíduos normais de mesma faixa etária, pela mastigação 


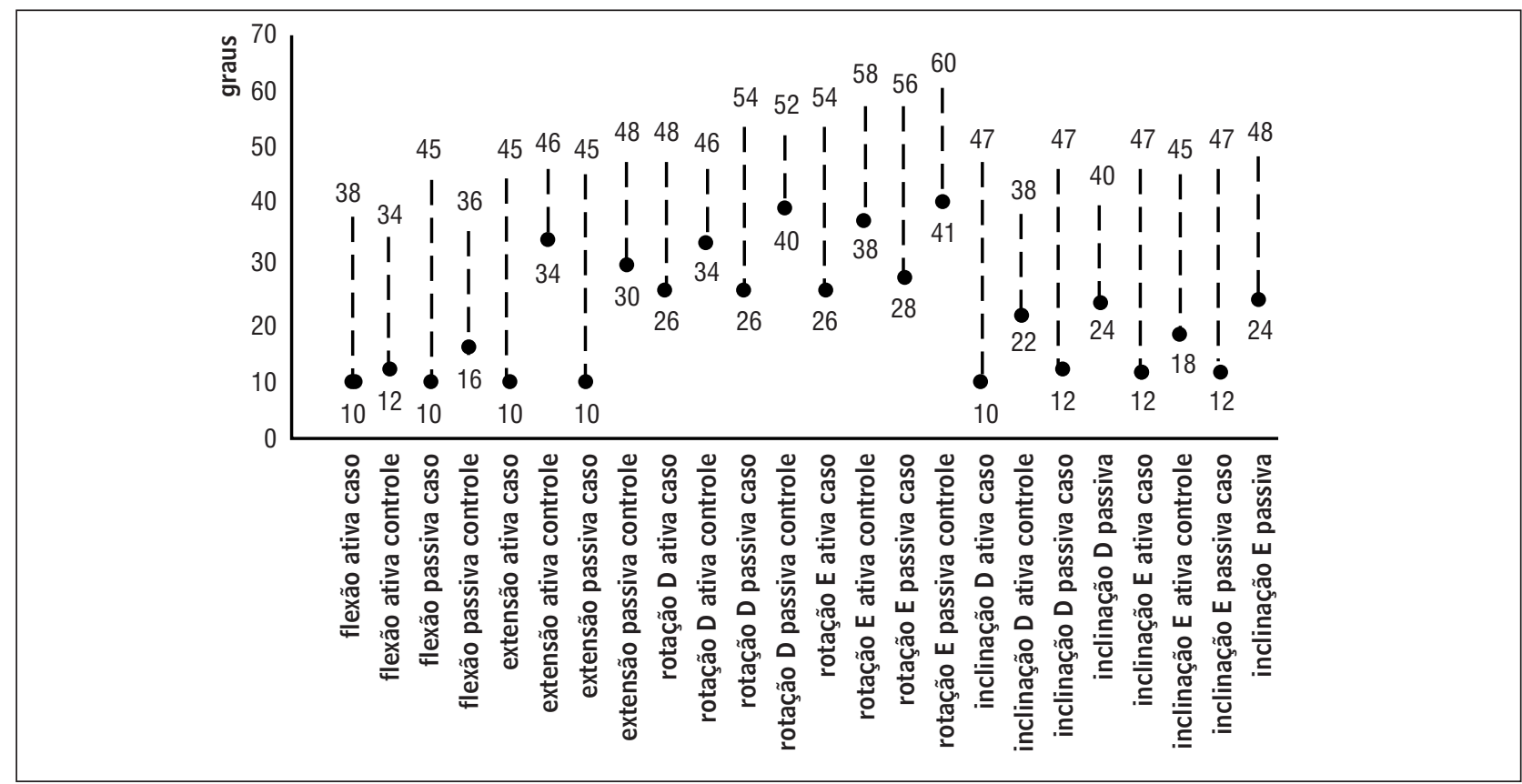

Gráfico 1 - Distribuição de máximos e mínimos de variação das avaliações médias goniométricas Fonte: Dados da pesquisa.

unilateral à esquerda. A unilateralidade mastigatória, mesmo que não exclusiva, além de prejudicar a performance mastigatória (13) prejudica, também, o desenvolvimento harmônico de estruturas envolvidas na mastigação, dependente da associação entre equilíbrio muscular e função (22). 0 movimento de qualidade da mandíbula durante a trituração do alimento é resultante dessa integração morfofuncional $(13,23)$.

Com a laringectomia total ocorrem alterações estruturais nos tecidos moles da região cervical, importantes para o processo da mastigação e para o suporte de cabeça e pescoço. Como desdobramento da traqueostomia, músculos da porção superficial e profunda (supra e infra-hioideos) são removidos - inclusive o osso hioideo, parcial ou totalmente (24), e ocorre manipulação na pele (5). Tanto a biomecânica do sistema estomatognático quanto a sustentação e amplitude de movimento de cabeça e pescoço estão afetadas $(3,4,25)$, seja por disfunção muscular ou óssea ou pelas sequelas do procedimento, como aderências e retrações, edema e dor que ocorrem na região cervical anterior (11, 24). Talvez por essas razões, o movimento mais comprometido em pacientes que se submeteram ao tratamento foi o de extensão por causa da manipulação intensa da região anterior do pescoço, interferindo em sua flexibilidade e atuando como uma barreira mecânica ao movimento, o qual depende não só de articulações saudáveis, mas também de tecidos conectores íntegros.

O movimento de extensão de cabeça é realizado pela contração concêntrica dos músculos posteriores de pescoço e da contração excêntrica da musculatura anterior da mesma região, ao passo que a atuação oposta acontece na flexão de cabeça (26).

Assim, o movimento de flexão também é atingido pela laringectomia total - mesmo não tendo sido encontrados resultados de significância estatística. 0 equilíbrio entre musculatura anterior e posterior de pescoço é prejudicado, produzindo aumento de tensão da musculatura de suporte posterior de cabeça e pescoço como um padrão compensatório para que a queda anterior da cabeça seja evitada e as funções vitais dependentes do posicionamento cefálico ereto sejam mantidas.

Para explicar a correlação entre um tipo de mastigação e a mobilidade de cabeça e pescoço, alguns estudos suportam a ideia de ligação funcional entre as regiões temporomandibular e craniocervical (14, $15,26)$; nesse caso, entende-se que os movimentos funcionais da mandíbula ocorrem em paralelo com movimento de cabeça, ou seja, que a movimentação funcional mandibular consiste de movimentos concomitantes de mandíbula e de cabeça e pescoço. Envolvem as articulações temporomandibular, 
Tabela 3 - Distribuição de médias, intervalos de confiança e intervalo de variação das avaliações goniométricas de laringectomizados e controles com preferência mastigatória à direita

\begin{tabular}{|c|c|c|c|c|c|c|c|c|}
\hline \multirow[b]{2}{*}{ Parâmetro } & & \multicolumn{3}{|c|}{ Casos com preferência de mordida à direita } & \multicolumn{3}{|c|}{$\begin{array}{l}\text { Controles com preferência de mordida à } \\
\text { direita }\end{array}$} & \multirow[b]{2}{*}{$\mathrm{p}$} \\
\hline & & $\begin{array}{l}\text { Média } \pm \text { Erro } \\
\text { padrão }\end{array}$ & IC95\% & Variação & $\begin{array}{l}\text { Média } \pm \text { Erro } \\
\text { padrão }\end{array}$ & IC95\% & Variação & \\
\hline \multicolumn{9}{|l|}{ Flexão } \\
\hline Ativa & & $21,00 \pm 6,03$ & $1,82-40,18$ & $10-38$ & $26,00 \pm 2,45$ & $18,20-33,80$ & $22-32$ & 0,245 \\
\hline Passiva & & $24,00 \pm 6,88$ & $2,11-45,89$ & $14-44$ & $30,50 \pm 1,71$ & $25,06-35,94$ & $26-34$ & 0,248 \\
\hline \multicolumn{9}{|l|}{ Extensão } \\
\hline Ativa & & $18,50 \pm 2,45$ & $9,00-28,00$ & $10-24$ & $38,00 \pm 4,55$ & $23,53-52,47$ & $26-46$ & $0,020^{*}$ \\
\hline Passiva & & $19,50 \pm 3,20$ & $9,31-29,69$ & $10-24$ & $41,50 \pm 4,27$ & $27,90-55,10$ & $30-48$ & $0,019^{*}$ \\
\hline \multicolumn{9}{|l|}{ Rotação } \\
\hline \multirow{2}{*}{ à direita } & ativa & $35,50 \pm 4,42$ & $21,42-49,58$ & $26-44$ & $42,00 \pm 1,87$ & $36,05-47,95$ & $37-46$ & 0,309 \\
\hline & passiva & $38,50 \pm 5,85$ & $19,88-57,12$ & $26-52$ & $45,25 \pm 1,25$ & $41,27-49,23$ & $42-48$ & 0,386 \\
\hline \multirow{2}{*}{ à esquerda } & ativa & $36,50 \pm 5,12$ & $20,19-52,81$ & $26-48$ & $49,50 \pm 3,20$ & $39,31-59,69$ & $40-59$ & 0,081 \\
\hline & passiva & $39,50 \pm 4,88$ & $24,27-54,73$ & $28-50$ & $52,25 \pm 3,84$ & $40,04-64,46$ & $41-58$ & 0,083 \\
\hline \multicolumn{9}{|l|}{ Inclinação } \\
\hline \multirow{2}{*}{ à direita } & ativa & $31,75 \pm 6,98$ & $9,53-53,97$ & $18-47$ & $28,50 \pm 3,59$ & $17,06-39,94$ & $22-38$ & 0,885 \\
\hline & passiva & $32,75 \pm 6,97$ & $10,57-54,93$ & $18-47$ & $30,00 \pm 3,56$ & $18,67-41,33$ & $24-40$ & 0,885 \\
\hline \multirow{2}{*}{$\begin{array}{l}\text { à } \\
\text { esquerda }\end{array}$} & ativa & $29,25 \pm 8,44$ & $239-56,11$ & $12-47$ & $27,50 \pm 2,87$ & $18,36-36,64$ & $24-36$ & 1,000 \\
\hline & passiva & $30,25 \pm 8,45$ & $3,36-57,14$ & $12-47$ & $30,50 \pm 2,63$ & $22,13-38,87$ & $26-38$ & 1,000 \\
\hline
\end{tabular}

Legenda: ${ }^{*}=$ estatisticamente significante.

Fonte: Dados da pesquisa

atlantoccipital e de coluna cervical, e são determinados pela ação conjunta entre músculos mandibulares e do pescoço $(15,26)$. Durante a depressão mandibular ocorre a extensão de cabeça e pescoço e a flexão ocorre durante a elevação da mandíbula. Isso supõe a existência de conexões neurais entre os sistemas sensório-motores cervical e trigeminal (26).

Douglas e Oncins (27) relatam, ainda, que as estruturas envolvidas na biomecânica da mastigação também servem como origem ou inserção para músculos envolvidos no suporte de cabeça e pescoço, gerando integração entre estruturas como mandíbula, crânio, hioide, esterno e coluna cervical.

Isso corrobora com o pensamento de que estímulos sensório-motores mandibulares podem influenciar no comportamento motor de cabeça e pescoço durante a mastigação (15).
Essas relações puderam ser evidenciadas, neste estudo, por meio da avaliação da amplitude de movimento do segmento cervical, que se mostrou reduzida em todos os movimentos analisados nos indivíduos laringectomizados totais. Entretanto, somente o movimento de extensão de cabeça, tanto de forma ativa quanto na forma passiva de avaliação, mostrou-se significativamente alterado nesta população em relação aos controles.

Em adição, quando a preferência mastigatória à esquerda foi comparada com os achados goniométricos, outro movimento que se mostrou limitado em laringectomizados totais foi o de inclinação para o lado direito, tanto na forma passiva quanto na ativa. Com esses achados, fica fortalecida a ideia de interferência entre alterações estruturais geradas por estímulos continuados em um lado da mandíbula, com maior ativação da 
Tabela 4 - Distribuição de médias, intervalos de confiança e intervalo de variação das avaliações goniométricas de casos e controles com preferência mastigatória à esquerda

\begin{tabular}{|c|c|c|c|c|c|c|c|c|}
\hline \multirow[b]{2}{*}{ Parâmetros } & & \multicolumn{3}{|c|}{$\begin{array}{c}\text { Casos com preferência de mordida à } \\
\text { esquerda }\end{array}$} & \multicolumn{3}{|c|}{$\begin{array}{l}\text { Controles com preferência de mordida à } \\
\text { esquerda }\end{array}$} & \multirow[b]{2}{*}{$\mathbf{P}$} \\
\hline & & $\begin{array}{l}\text { Média } \pm \\
\text { Erro padrão }\end{array}$ & IC95\% & Variação & $\begin{array}{l}\text { Média } \pm \\
\text { Erro padrão }\end{array}$ & IC95\% & Variação & \\
\hline \multicolumn{9}{|l|}{ Flexão } \\
\hline Ativa & & $20,80 \pm 4,92$ & $7,13-34,47$ & $10,00-32,00$ & $23,20 \pm 3,98$ & $12,15-34,25$ & $12,00-34,00$ & 0,599 \\
\hline Passiva & & $22,00 \pm 4,94$ & $8,29-35,71$ & $10,00-34,00$ & $26,80 \pm 4,08$ & $15,47-38,13$ & $16,00-36,00$ & 0,346 \\
\hline \multicolumn{9}{|l|}{ Extensão } \\
\hline Ativa & & $23,00 \pm 5,83$ & $6,81-39,19$ & $10,00-45,00$ & $41,20 \pm 1,62$ & $36,69-45,71$ & $36,00-46,00$ & 0,071 \\
\hline Passiva & & $24,20 \pm 5,80$ & $8,10-40,30$ & $10,00-45,00$ & $43,60 \pm 1,72$ & $38,82-48,38$ & $38,00-48,00$ & $0,047^{*}$ \\
\hline \multicolumn{9}{|l|}{ Rotação } \\
\hline \multirow{2}{*}{ à direita } & ativa & $38,80 \pm 3,61$ & $28,77-48,83$ & $26,00-48,00$ & $40,20 \pm 1,96$ & $34,76-45,64$ & $34,00-46,00$ & 0.916 \\
\hline & passiva & $42,80 \pm 4,50$ & $30,31-55,29$ & $28,00-54,00$ & $45,20 \pm 1,96$ & $39,76-50,64$ & $40,00-52,00$ & 0,674 \\
\hline \multirow{2}{*}{ à esquerda } & ativa & $48,00 \pm 2,68$ & $40,55-55,45$ & $38,00-54,00$ & $48,20 \pm 3,20$ & $39,32-57,08$ & $38,00-58,00$ & 0,750 \\
\hline & passiva & $50,40 \pm 2,99$ & $42,09-58,71$ & $40,00-56,00$ & $52,80 \pm 2,42$ & $46,09-59,51$ & $46,00-60,00$ & 0,671 \\
\hline \multicolumn{9}{|l|}{ Inclinação } \\
\hline \multirow{2}{*}{ à direita } & ativa & $21,60 \pm 3,31$ & $12,41-30,79$ & $10,00-30,00$ & $30,20 \pm 1,91$ & $24,90-35,50$ & $24,00-34,00$ & $0,044^{*}$ \\
\hline & passiva & $22,80 \pm 3,20$ & $13,92-31,68$ & $12,00-32,00$ & $32,80 \pm 1,62$ & $28,29-37,31$ & $28,00-38,00$ & $0,027^{*}$ \\
\hline \multirow{2}{*}{ à esquerda } & ativa & $26,40 \pm 2,99$ & $18,09-34,71$ & $20,00-36,00$ & $31,40 \pm 4,47$ & $19,00-43,80$ & $18,00-45,00$ & $0,395^{*}$ \\
\hline & passiva & $28,00 \pm 2,68$ & $20,55-35,45$ & $22,00-36,00$ & $35,60 \pm 3,20$ & $24,72-46,48$ & $24,00-48,00$ & $0,092^{*}$ \\
\hline
\end{tabular}

Legenda: * = estatisticamente significante.

Fonte: Dados da pesquisa.

Nota: Valores de p calculados com o teste não paramétrico de Mann-Whitney para comparação de médias de amostras não pareadas.

musculatura mastigatória, produzindo mudanças na tensão muscular nos músculos responsáveis pelo suporte de cabeça do mesmo lado $(28,29)$.

Outro dado, em particular, que merece atenção é que, mesmo naqueles indivíduos laringectomizados totais, com predominância pelo lado direito na mastigação, a extensão de cabeça apresentou movimento com amplitude limitada.

Nota-se, então, que, além de todas as repercussões anatômicas e de função oriundas de uma laringectomia total, o prejuízo à mobilidade da região adjacente ao local de procedimento, principalmente para a extensão de cabeça, é real, o que releva a atuação mais direcionada à manutenção ou recuperação da amplitude de movimento da coluna cervical. Além disso, é importante investir na avaliação de outras possíveis alterações que possam amplificar sequelas musculoesqueléticas, como a mastigação unilateral.

\section{Conclusão}

Neste estudo observou-se que, em laringectomizados totais, a preferência por lado mastigatório ocorre à esquerda, sendo acompanhada de redução da amplitude de movimento da extensão passiva e da inclinação para a direita, tanto ativa quanto passiva, apesar de serem encontradas limitações em todos os demais movimentos analisados. Ainda assim, são necessários outros estudos com o intuito de estratificar os achados com relação às variáveis idade e gênero dos voluntários. 


\section{Agradecimentos}

Ao CNPq - Edital MCT/CNPq n. 70/2009 Mestrado/Doutorado; Edital MCT/CNPq 14/2009Universal - Faixa B.

\section{Referências}

1. Herranz J, Sarandeses A, Fernández MF, Barro CV, Vidal JM, Gavlán J. Complications after total laryngectomy in nonradiated laryngeal and hypopharyngeal carcinomas. Otolaryngol Head Neck Surg. 2000;122(6):892-8.

2. Braz DSA, Ribas MM, Dedivitis RA, Nishimoto IM, Barros APB. Quality of life and depression in patients undergoing total and partial laryngectomy. Clinics. 2005;60(2):135-42.

3. Hannickel S, Zago MMF, Barbeira CBS, Sawada NO. O comportamento dos laringectomizados frente à imagem corporal. Rev bras cancerol. 2002;48(3):333-9.

4. Barros APB, Portas JG, Queija DS, Lehn CN, Dedivitis RA. Autopercepção da desvantagem vocal (VHI) e qualidade de vida relacionada à deglutição (SWAL-QDL) de pacientes laringectomizados totais. Rev Bras Cirurg Cab Pesc. 2007;36(1):32-7.

5. Freitas TA, Lynch CS, Machado e Silva H. Câncer de laringe e fonoaudiologia. Lato \& Sensu. 2003;4(1):3-5.

6. Bettinelli LA, Tourinho H Filho, Capoani P. Experiências de idosos após laringectomia total. Rev Gaúcha Enferm. 2008;29(2):214-20.

7. Paula FC, Gama RR. Avaliação de qualidade de vida em laringectomizados totais. Rev Bras Cirurg Cab Pesc. 2009;38(3):177-82.

8. Lo Tempio MM, Wang KH, Sadeghi A, Delacure MD, Juillard GF, Wang MB. Comparison of quality of life outcomes in laryngeal câncer patients following chemoradiation vs. total laryngectomy. Otolaryngol Head Neck Surg. 2005;132(6):948-53.

9. Chen AY, Schrag N, Hao Y, Flanders WD, Kepner J, Steward A, et al. Changes in treatment of advanced laryngeal cancer 1985-2001. Otolaryngol Head Neck Surg. 2006;135(6):831-7.

10. Aprigliano F, Mello LFP. Tratamento cirúrgico do câncer da laringe - análise de 1055 casos. Arq Int Otorrinolaringol. 2006;10(1):36-45.
11. Santos CB, Caria PHF, Tosello DO, Bérzin F. Comportamento dos músculos cervicais em indivíduos com fala esofágica e laringe artificial. Rev CEFAC. 2010;12(1):82-90.

12. Pastana SG, Costa SM, Chiappetta ALML. Análise da mastigação em indivíduos que apresentam mordida cruzada unilateral na faixa-etária de 07 a 12 anos. Rev CEFAC. 2007;9(3):339-50.

13. Gomes SGF, Custódio W, Jufer JSM, Cury AADB, Garcia RCMR. Correlation of mastication and masticatory movements and effect of chewing side preference. Braz Dent J. 2010;21(4):351-5.

14. Ries LGK, Alves MC, Bérzin F. Asymmetric activation of temporalis, masseter, and sternocleidomastoid muscles in temporomandibular disorder patients. Cranio. 2008;26(1):59-64.

15. Häggman-Henrikson B, Eriksson PO. Head movements during chewing: relation to size and texture of bolus. J Dent Res. 2004;83(11):864-68.

16. Andrade JA, Leite VM, Salmela LFT, Araujo PMP, Juliano Y. Estudo comparativo entre os métodos de estimativa visual e goniometria para avaliação das amplitudes de movimento da articulação do ombro. Acta fisiatr. 2003;10(1):12-6.

17. Venturni C, André A, Aguilar BP, Giacomelli B. Confiabilidade de dois métodos de avaliação da amplitude de movimento ativa de dorsiflexão do tornozelo em indivíduos saudáveis. Acta fisiatr. 2006;13(1):41-5.

18. Sacco ICN, Alibert S, Queiroz BWC, Pripas D, Kieling I, Kimura AA, et al. Confiabilidade da fotogrametria em relação a goniometria para avaliação postural dos membros inferiores. Rev bras fisioter. 2007;11(5):411-17.

19. Nascimento GKBO. Força de mordida e atividade elétrica dos músculos masseter e temporal anterior em laringectomizados [dissertação]. Recife: Universidade Federal de Pernambuco; 2010.

20. Moraes KJRM. Análise eletromiográfica do esternocleidomastóideo e do trapézio superior e suas repercussões na postura e no pós-operatório de laringectomia total [dissertação]. Recife: Universidade Federal de Pernambuco; 2010.

21. Taboadela CH. Goniometría: uma herramienta para la evaluación de las incapacidades laborales. 1. ed. Buenos Aires: Asociart ART; 2007. 
22. Munõz GC, Carvalho CS, Misaki JK, Gomes ICD, Carvalho ARR. Análise dos potenciais elétricos do músculo masseter durante a mastigação de alimentos com rigidez variada. Rev CEFAC. 2004;6(2):127-34.

23. Berretin-Felix G, Araújo ES. Fisiologia da contração do músculo esquelético e do exercício aplicada à motricidade orofacial. In: Silva HJ, Cunha DA. O sistema estomatognático: anatomofisiologia e desenvolvimento. São José dos Campos: Pulso Editorial; 2011. p. 72-82.

24. Marszaiek S, Zebryk-Stopa A, Krasny J, Obrebowski A, Golusinski W. Estimation of influence of myofascial release techniques on esophageal pressure in patients after total laryngectomy. Eur Arch Otorhinolaryngol. 2009;266(8):1305-8.

25. Oliveira IB, Servilha BB, Ferreira LA, Bastos TS, Freire VO, Chagas JFS. Qualidade de vida de pacientes pós-cirúrgicos de tumores malignos da cabeça e pescoço. Rev Ciênc Méd. 2005;14(6):523-8.

26. Zafar H, Nordh E, Eriksson PO. Temporal coordination between mandibular and head-neck movements during jaw opening-closing tasks in man. Arch Oral Biol. 2000;45:675-82.
27. Douglas CR, Oncins MC. Fisiologia geral do sistema estomatognático. In: Silva HJ, Cunha DA. 0 sistema estomatognático: anatomofisiologia e desenvolvimento. São José dos Campos: Pulso Editorial; 2011. p. 36-57.

28. Yi LC, Guedes ZCF,Vieira MM. Relação da postura corporal com a disfunção da articulação temporomandibular: hiperatividade dos músculos da mastigação. Fisioter Bras. 2003;4(5):341-7.

29. Shimazaki K, Matsubara N, Hisano M, Soma K. Functional relationships between the masseter and sternocleidomastoid muscle activities during gum chewing: the effect of experimental muscle fatigue. Angle Orthod. 2006;76(3):452-8.

Recebido: 20/05/2012

Received: 05/20/2012

Aprovado: 19/12/2012

Approved: 12/19/2012 